\title{
Optical polarimetry and molecular line studies of L1157 dark molecular cloud
}

\author{
Ekta Sharma ${ }^{1 *}$ Archana Soam ${ }^{2}$, Maheswar Gopinathan ${ }^{1}$ \\ ${ }^{1}$ Aryabhatta Research Institute of Observational Sciences (ARIES), Nainital 263002, India \\ ${ }^{2}$ Korea Astronomy \& Space Science Institute (KASI), 776 Daedeokdae-ro, Daejeon, \\ Republic of Korea
}

\begin{abstract}
Filaments are omnipresent in molecular clouds which are believed to fragment into cores. The detailed process of the evolution from filaments to cores depends critically on the physical conditions in the star forming region. This study aims at characterising gas motions using velocity structure and finding the dynamical importance of magnetic fields in the filament morphology. The plane-of-the-sky component of the magnetic field has been measured using optical polarization of the background stars. The orientation is found to be almost perpendicular to the filament implying its dynamical importance in the evolution of the cloud. Optical polarimetric results match very well with the sub millimetre polarization angles obtained in the inner core regions. The magnetic fields are found to have an orientation of $130^{\circ}$ east with respect to north. The angular offset between the outflow axis and the magnetic field direction is found to be $25^{\circ}$. Values for parameters like the excitation temperature, optical depth and column densities have been derived using molecular lines. Optically thick lines show non-gaussian features. The non-thermal widths tell about the presence of turbulent motions whereas the $\mathrm{C}^{18} \mathrm{O}$ lines follow Gaussian features almost at all the locations observed in the filament.
\end{abstract}

\section{Introduction}

Recent Herschel observations (Andre et al. 2010) of nearby dusty clouds have shown that filamentary structures are ubiquitous in molecular clouds following the bead-string scenario. Cores are believed to form in molecular clouds by fragmentation of filaments that might arise by gravitational instabilities or turbulence. Numerical simulations involving turbulence and self gravity also have shown that filaments are always present on all scales in the interstellar medium (ISM) and gas compresses out of the sheets and filaments (Vazquez-Semadeni 1994, Padoan et al. 2001, Ostriker et al. 1999, Ballesteros-Paredes et al. 1999). Magnetic fields (B-fields) also play an important role in determining the shape of the filament as the material tends to move along the field lines giving rise to the flattened structure (Galli \& Shu 1993). This type of structure has been confirmed from ambipolar diffusion models (Fiedler \& Mouschovias 1993). Dichroic extinction of background starlight by magnetically aligned dust particles at the periphery of dark molecular clouds introduces polarization in the starlight (Davis \& Greenstein 1951) which is used to determine the plane-of-the-sky component of the B-field.

*ektasharma@aries.res.in 
In this work, we have studied the source L1157 dark cloud (Lynds 1966). It harbours L1157-IRS or IRAS 20386+6751: a class 0 protostar and a well collimated bipolar outflow with a spatial size of 5 arcmin. This object is in the transition phase from cloud collapse to form star formation. Because of the different molecule chemistry at various layers of the cloud, tracers with different critical densities are used to determine the whole kinematics. We have mapped the B-field in the optical regime and observed four molecular lines $\left({ }^{12} \mathrm{CO}, \mathrm{C}^{18} \mathrm{O}, \mathrm{HCN}, \mathrm{N}_{2} \mathrm{H}^{+}\right)$along the whole filament.

\section{Observations}

\subsection{Optical Polarimetry}

The polarimetric observations for the dark cloud were carried out at cassegrain focus of the 1.04-m Sampurnanand Telescope using the Aries IMaging POLarimeter (AIMPOL) at the Aryabhatta Research Institute of Observational SciencES (ARIES; Nainital, India). The plate scale and gain of the CCD used are 1.48 arcsec per pixel and $11.98 \mathrm{e}^{-}$per ADU (Analog to digital unit). The noise created during CCD read-out is $7.0 \mathrm{e}^{-}$. The standard Johnson $\mathrm{R}_{k c}$ filter with $\lambda_{\text {eff }}$ as $0.76 \mu \mathrm{m}$ was used for polarimetric observations. The instrumental polarization derived from unpolarized standards is found out as $0.1 \%$.

\subsection{Radio Observations}

The filamentary structure of the $\mathrm{L} 1157$ cloud has been mapped in ${ }^{12} \mathrm{CO}, \mathrm{C}^{18} \mathrm{O}, \mathrm{HCN}, \mathrm{N}_{2} \mathrm{H}^{+}$lines using the 13.7-m diameter single dish radio facililty at the Taeudek Radio Astronomy Observatory (TRAO) which is located at Korea Astronomy and Space Science Institute (KASI; Daejeon,South Korea). It operates in the wavelength range of $85-135 \mathrm{GHz}$. The pointing accuracy is 0.5 " and the $\mathrm{SiO}$ line of the standard source Orion A was used to focus the telescope. At $115 \mathrm{GHz}$, the beam size of the telescope (HPBW) is about $44^{\prime \prime}$ while the fraction of the beam pattern subtending the main beam (beam efficiency) is $54 \pm 2 \%$. The system temperature was $550-600 \mathrm{~K}$ during observations. The integration time of On-The-Fly (OTF) mapping was around 180 minutes to achieve a root-meansquare (rms) of $0.4 \mathrm{~K}$ in peak antenna temperature $\left(T_{A}{ }^{*}\right)$ scale in $\mathrm{CO}$ line. The signal-to-noise ratio (SNR) is measured to be 16 with the peak $T_{A}{ }^{*}$ as $4.8 \mathrm{~K}$. The achieved velocity resolution is $\sim 0.16$ $\mathrm{kms}^{-1}$.

\section{Results}

Fig. 1 shows the $\mathrm{R}_{k c}$ band polarization vectors overplotted on the $12 \mu \mathrm{m}$ image of $0.3^{\circ} \times 0.3^{\circ}$ obtained with the Wide-Field Infrared Survey Explorer (WISE) ${ }^{1}$. The angle of polarization has been measured with respect to the north-south axis towards east. Each vector in the map represents the measurement with SNR ratio, $\mathrm{P} / \sigma_{p} \geq 2$. The mean values of the degree of polarization $(\mathrm{P})$ and the polarization angle $\left(\theta_{p}\right)$ with their errors (from Gaussian fitting) are found to be $2.6 \pm 0.6 \%$ and $130 \pm 12^{\circ}$, respectively. Using the modified Chandrashekhar-Fermi (CF) relation (Chandrashekhar \& Fermi 1953), the B-field strength towards LDN 1157 has been calculated as $86.5 \mu \mathrm{G}$.

Fig. 2 shows the integrated intensity contour maps of ${ }^{12} \mathrm{CO}(\mathrm{J}=1-0), \mathrm{C}^{18} \mathrm{O}(\mathrm{J}=1-0), \mathrm{HCN}(\mathrm{J}=1-0)$ and $\mathrm{N}_{2} \mathrm{H}^{+}(\mathrm{J}=1-0)$ overplotted on the $12 \mu \mathrm{m}$ WISE image showing the L1157 cloud observed in a region of $8^{\prime} \times 8^{\prime}$.

\footnotetext{
${ }^{1}$ www.skyview.gsfc.nasa.gov
} 


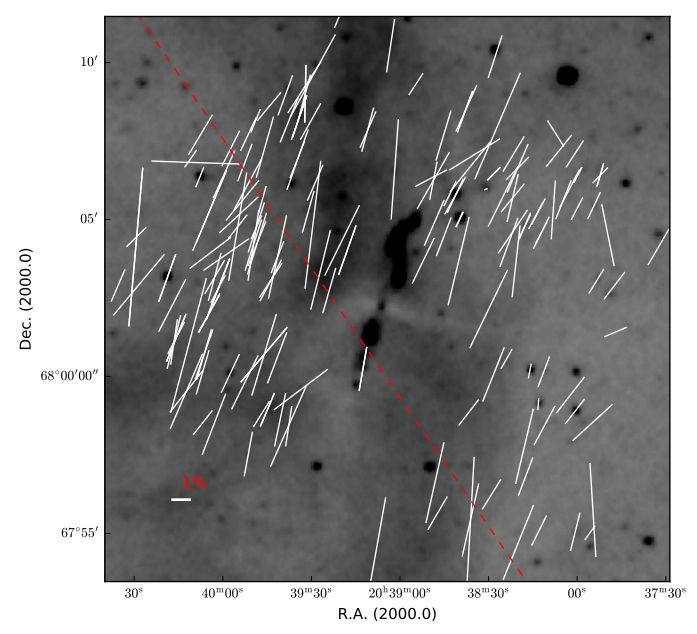

Figure 1: Optical polarization vectors over-plotted on the $0.3^{\circ} \times 0.3^{\circ}$ WISE $12 \mu \mathrm{m}$ image of LDN 1157. The red dashed line shows the direction of the galactic plane.

\section{Discussions and Conclusions}

The ubiquity of filaments and their theoretical studies make it essential to understand the evolution of filaments. They are believed to be the birth sites of cores in dark clouds. Herschel images observed at a wavelength of $250 \mu \mathrm{m}$ clearly show that the filaments are extended in the east-west direction with a second core emerging in the south of the source.

The B-field morphology in the outer regions of the cloud inferred from optical polarimetry is found to be similar to that of inner region measured using sub-mm polarization (Stephens et al. 2014). Considering outflow as a proxy of the rotation axis, its orientation can be compared with the B-field orientation. There is an angular offset of $25^{\circ}$ between the envelope B-field direction and the outflow position angles. It is almost parallel to the hour glass axis exhibited by the inner B-field. The observed offset between the outflow (a proxy of the rotation axis) and the B-field could be responsible for the formation of the rotationally supported disk around the protostar (Hull et al. 2014). The results stated here show the dynamical importance of the B-field in the evolution of this dark cloud. Polarimetry in near-infrared (NIR) wavelengths can trace the geometry of relatively high density inner regions of the cloud. So, a NIR polarimeter attached to the 3.6-m Devesthal Optical Telescope (DOT) would help in getting complete information about the field orientation of these kind of objects.

The kinematics of inhomogeneous gas in the whole filament have been inferred using the velocity structure of different tracers. ${ }^{12} \mathrm{CO}$ traces most of the envelope while the high-density tracers such as $\mathrm{C}^{18} \mathrm{O}$ traces only the denser parts in the envelope of L1157. The infall features and the presence of bipolar outflows in the cloud are indicated by the line shapes in the $\mathrm{CO}$ spectra. As seen from the integrated intensity contours, $\mathrm{N}_{2} \mathrm{H}^{+}$and $\mathrm{HCN}(\mathrm{J}=1-0)$ being less depleted, are effectively tracing the high density and cold regions. $\mathrm{CO}$ lines are highly asymmetric with a relatively higher value of antenna temperature whereas the $\mathrm{C}^{18} \mathrm{O}$ emission has a symmetric distribution around the local standard of rest velocity $\left(\mathrm{V}_{l s r}\right)$. This suggests that there could be different sub-structures with a varying velocity in the envelope which could reduce to the single static component in the dense core regions as inferred by $\mathrm{C}^{18} \mathrm{O}$ line profiles. The presence of the protostar in the cloud marks the onset of the basic processes of star formation. The line width gives information about the thermal and non-thermal motion of the material. The variation in velocity dispersion along the filament leads to conclusions about the kinematics. The velocity dispersion of the spectra around the central source 

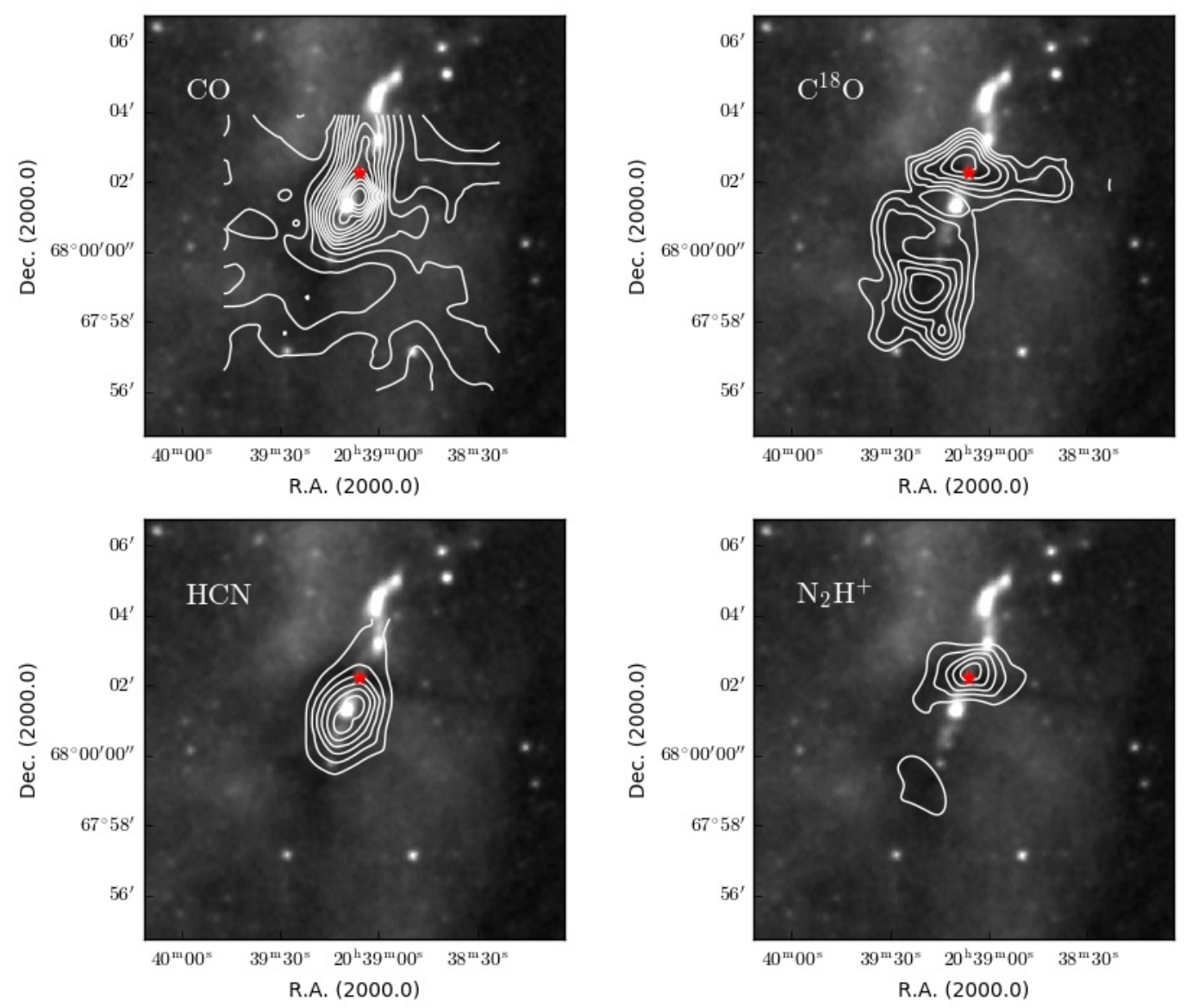

Figure 2: Integrated intensity contours for ${ }^{12} \mathrm{CO}, \mathrm{C}^{18} \mathrm{O}, \mathrm{HCN}, \mathrm{N}_{2} \mathrm{H}^{+}$overplotted on $12 \mu \mathrm{m}$ WISE $0.5^{\circ} \times 0.5^{\circ}$ image. The contour levels range from 3 to $17.9 \mathrm{~K} \mathrm{kms}^{-1}$ in steps of $1.0 \mathrm{~K} \mathrm{kms}^{-1}$ for ${ }^{12} \mathrm{CO}$ ( $\mathrm{J}=1-0$ ) (top left), from 0.35 to $0.63 \mathrm{~K} \mathrm{kms}^{-1}$ in steps of $0.05 \mathrm{~K} \mathrm{kms}^{-1}$ for $\mathrm{C}^{18} \mathrm{O}(\mathrm{J}=1-0)$ (top right), from 1.0 to $5.12 \mathrm{~K} \mathrm{kms}^{-1}$ in steps of $0.8 \mathrm{~K} \mathrm{kms}^{-1}$ for $\mathrm{HCN}(\mathrm{j}=1-0)$ (bottom left), and from 0.5 to 1.47 $\mathrm{K} \mathrm{kms}^{-1}$ in steps of $0.2 \mathrm{~K} \mathrm{kms}^{-1}$ for $\mathrm{N}_{2} \mathrm{H}^{+}(\mathrm{J}=1-0)$ (bottom right). The red star marks the presence of the central protostars. The contour levels are in the units of $\mathrm{K} \mathrm{kms}^{-1}$.

is found to be greater than $0.1 \mathrm{kms}^{-1}$, characterising turbulent motions. These preliminary results highlight the importance of factors like turbulence and B-field in the dynamics of the dark cloud.

\section{Acknowledgements}

We thank the observing staff and observing assistants at the 1.04-m Sampurnanand Telescope. We also acknowledge the use of NASA's SkyView facility (http://skyview.gsfc.nasa.gov) located at the NASA Goddard Space Flight Center. 


\section{References}

André P., Menshchikov A., Bontemps S. et al. 2010, A\&A, 518, L102

Ballesteros-Paredes J., Vzquez-Semadeni E., Scalo J. 1999, ApJ, 515, 286

Davis L. J., Greenstein J. L. 1951, ApJ, 114, 206

Fiedler R. A., Mouschovias T. C. 1993, ApJ, 415, 680

Galli D., Shu F. H. 1993, ApJ, 417, 220

Hull C. L. H., Plambeck R. L., Bolatto A. D. et al. 2013, ApJ, 768, 159

Ostriker E. C., Gammie C. F., Stone J. M. 1999, ApJ, 513, 259

Padoan P., Juvela M., Goodman A. A., Nordlund . 2001, ApJ, 553, 227

Rautela B. S., Joshi G. C., Pandey J. C. 2004, BASI, 32, 159

Stephens I. W., Looney L. W., Kwon W. et al. 2013, ApJ, 769, L15

Vazquez-Semadeni E. 1994, ApJ, 423, 681 